Books, videos, CD-ROMs, DVDs and any other relevant items submitted for a review in the $B D J$ should be addressed to: Kate Maynard, Assistant Editor, British Dental Journal, NPG, 4-6 Crinan Street, London N1 9XW

\section{Mastering digital dental photography}

\author{
W. Bengal \\ UK: Quintessence \\ price $€ 120$, pp 394 \\ ISBN 1850971528
}

First impressions are that this is a quality well produced and presented reference book on all aspects of digital dental photography. On further inspection these observations were confirmed: just looking through the index made the anticipation of the content exciting.

Split into three logical sections Technical, Practical and Digital workflow - the reader can easily jump to chapters of interest and detailed 'how to' instructions.

The Technical section comprises four chapters with explanations of digital cameras, lenses, lighting and capture technologies, well categorised for the novice to pick up salient points and to give the more experienced user a better understanding of the technology.

Moving on to the Practical section, this starts with a description of aids to get visual access to all areas in the mouth, namely using mirrors and retractors. A description of the standard intra oral and portrait views likely to be required follows, with associated recommended techniques. The methods described to achieve good portrait shots concentrated on studio lighting techniques, perhaps not 'practical' in most dental practices. Studio lighting techniques are also discussed in the chapter 'Photographing small objects'; this takes four chapters and gives good advice on the professional techniques employed, but sadly, little advice on techniques which can be used without the use of studio lighting. The last chapter in this section briefly covers digitising existing slides and prints.

'The digital workflow' section ie stages in the digital imaging process, is the largest section, twice the size of either of the other sections and for good reason.
Digital workflow directly affects your time and digital output, two of the most important elements in digital imaging. The section covers image transfer and includes a small section on RAW files, which I feel will emerge as the standard format in the future. This is followed by a few well documented chapters on image editing procedures covering topics such as image sharpening, treatment simulation, whitening teeth, red eye removal etc. Advanced procedures are also covered, creating collages, adding text content to your images etc. The final chapter is dedicated to slide presentations using PowerPoint, a very useful overview of what can be achieved in PowerPoint to present your prized images.

This is an excellent book of reference and practical procedures on all aspects of digital dental photography, and would be a valuable asset for both novice and experienced members of the dental team.

M. R. Sharland

\section{End stage renal disease: The oral component}

\section{P. Bots \\ The Netherlands: Optima Grafische Communicatie pp 152}

ISBN 9085591023

This is a very specialised book which is well written, but aimed at a fairly exclusive audience. The author is essentially a dentist who has a strong research interest in the oral complications of end stage renal disease (ESRD). There are virtually no previous similar publications, which makes this book unique.

The book is a slim, A5 soft back with many tables, but very few illustrations. There are 10 chapters in total. The first chapter covers a definition of ESRD, the role of saliva in oral health and the influence of ESRD on oral health. The following six chapters are individually dedicated to one study undertaken by the author and his research team.
The titles include 'The acute effects of haemodialysis on salivary flow rate and composition' and 'Oral and salivary changes in patients with end-stage renal disease: a two year follow-up study'. The next three chapters are dedicated to a general discussion, a summary in English and finally a summary in Dutch. Interestingly the appendix contains yet another study, a list of all the author's publications and his curriculum vitae. The final chapter is in Dutch and appears to be an afterthought thanking colleagues and friends.

Although this book is interesting to read it is presented in a scientific way with limited emphasis on clinical patient management. The general discussion and summary briefly cover some aspects of clinical patient management. These are difficult to recognise however, as there are no boxes or tables to highlight this information.

In summary I would recommend this book to all clinicians: dental, medical and surgical who manage patients with ESRD. Unfortunately however, although it does contain a lot of scientific material, it sometimes feels more like an anthology summarising the author's work to date, rather than a clear guide to the clinical management of these patients.

H. Zaitoun

\section{Fundamentals of operative dentistry}

\author{
J. B. Summit, J. W. Robbins, T. J. Hilton, \\ R. S. Schwartz \\ UK: Quintessence \\ price $€ 56.00$, pp 612 \\ ISBN 0867154527
}

This book is intended to be an in-depth reference work on contemporary practical operative dentistry, and as such has much to commend it. The authors aim to present a mix of traditional time proven methods together with modern clinical 

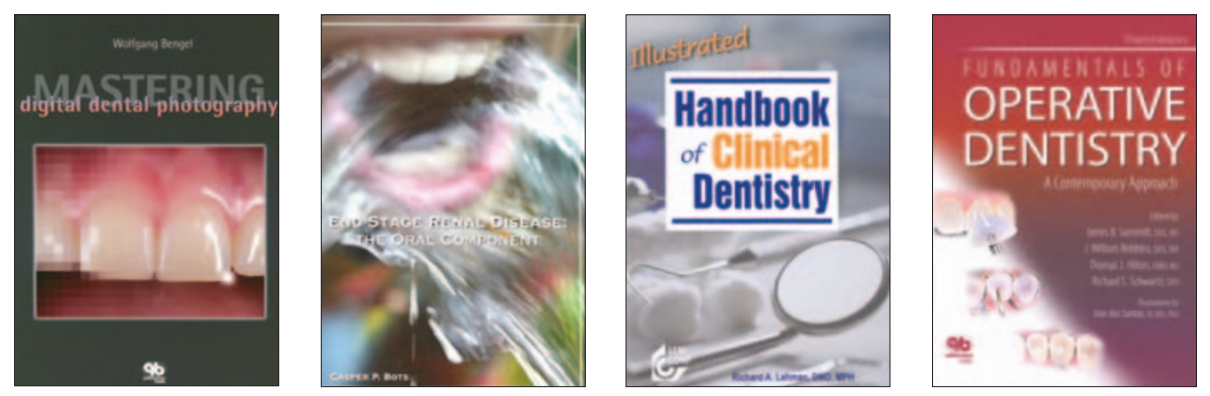

developments, and broadly succeed in this task. However, the content gives an impression of being slightly at odds with the title. Rather than a truly contemporary integrated approach to operative dentistry, the book's format and content is that of a more traditional text, with added sections of more modern relevance.

Overall the book is presented with a well ordered structure, and each chapter has a clearly defined field of reference. The sections begin with an in depth overview of the biological considerations underpinning operative dentistry, and progress logically through evaluation and clinical management before describing aspects of clinical care. Coverage of the field is exhaustive, including new adhesive techniques and tooth whitening, but some older procedures described in detail have less relevance to contemporary practice. Indeed, much space is devoted to techniques that have changed little in decades. Whilst this is not inappropriate, in some sections the inclusion of such procedures gives an old fashioned tone to the content, and this is where this book misses the opportunity to be truly modern.

Despite this, the subject matter is presented logically and clearly and all techniques are given a clear supporting background of both science and contributors' experience. The section on bonding to enamel and dentine is particularly worthy of note, as are the chapters devoted to composite resin restorations and anterior ceramic reconstructions. Illustrations are plentiful, appropriate, and of overall excellent quality, though there are many black and white clinical photographs which would have been clearer if presented in colour. The text is clear, delivered in an easily read style, and extensively referenced.

This is a valuable technical reference work, but there is much that could be omitted so that the content matches the philosophy of the title. The overall approach to operative dentistry is broadly mechanistic and many procedures described are of more historical interest rather than appropriate to current practice. As a manual of operative techniques it is a worthwhile resource, but rather than a truly contemporary review of the subject, it is grounded firmly in an established procedural approach to clinical matters. Nonetheless, this detracts little from the overall value of this edition as an excellent reference text.

A. J. Crothers

\section{Illustrated handbook of clinical dentistry}

\author{
R. A. Lehman \\ USA: Lexi-Comp \\ price $\$ 34.95$, pp 306 \\ ISBN 1930598521
}

This book is an easy to use reference manual aimed at dental undergraduates and general dental practitioners. It is well presented in an $\mathrm{A} 5$ soft back with wire binding. Titled chapter dividers make the book easy to navigate. The pages are thick and glossy, designed for ultimate longevity. In addition to the chapter dividers there is a comprehensive table of contents at the front of the book. Each chapter is attractively divided into subsections containing illustrations, highlighted tables and flow charts rather than solid text.

The book contains the following 14 chapters: Anaesthesia, Emergency medicine, Endodontics, Head and neck anatomy, Implantology, Instrumentation, Oral surgery, Orthodontics, Pathology, Paediatric dentistry, Periodontology, Prescription medications, Prosthodontics and radiology. The authors have various medical and dental qualifications, ensuring that all the major disciplines of clinical dentistry and emergency medicine are covered by a specialist in that field.

The Head and neck anatomy, Pathology, Periodontology and Radiology chapters are particularly good with a very concise summary of each of these subjects and appropriate tables and illustrations. Other chapters are not so consistent, although they do cover some of the basics, including lists and tables of those dental 'must knows' that are painfully hard to remember.

Another important point is that as this book is American the recommendations in the emergency medicine and infective endocarditis prophylaxis sections do vary from the UK guidelines.

In summary I would not recommend this book as a first choice for UK undergraduates, on the grounds that it is American and varies from UK guidelines. However, it will serve well as a quick reference book, and would be a good friend during preparation for exams.

H. Zaitoun 